\title{
Los grados del diminutivo: una doctrina confusa en la gramática latina antigua*
}

\author{
Julia Burghini y Javier Uría \\ Universidad Nacional de Córdoba - CONICET \\ juliburghini@gmail.com \\ ORCID iD: http://orcid.org/0000-0003-3754-3159 \\ Universidad de Zaragoza \\ juria@unizar.es \\ ORCID iD: http://orcid.org/0000-0001-6386-1789
}

\section{The degrees of the diminutive: a confused doctrine in the ancient Latin grammar}

\begin{abstract}
Desde Varrón a Prisciano, los gramáticos latinos se interesaron por la acumulación de los sufijos diminutivos (-ulus, -culus, etc.), relacionándola con frecuencia con los grados de comparación $\mathrm{y}$, por ende, con un aumento de la función diminutiva. En las gramáticas latinas antiguas, el tratamiento de los diminutivos era heterogéneo: desde una perspectiva semántica se destacaba su cercanía a los grados de comparación -en tanto representan la disminución de la esencia de un nombre- y desde una formal se clasificaban como derivados -en tanto se derivan de nombres primitivos-. Aunque la mayoría de los gramáticos relaciona el diminutivo con el comparativo y el derivativo, existen
\end{abstract}

From Varro to Priscian, Latin grammarians paid close attention to the accumulation of diminutive suffixes (-ulus, -culus, etc.), often relating it to the degrees of comparison and therefore to an increase in diminutive function. The treatment of diminutives in ancient Latin grammars handbooks was not uniform: from a semantic point of view, they were related to the degrees of comparison (i.e. they represent the diminution of the essence of a noun); and from a formal point of view, they were classified as derivatives (i.e., they derived from primitive names). Although most grammarians relate the diminutive to the comparative and the derivative, there are significant differences in

* Un avance parcial de este artículo fue presentado por J. Burghini en las VIII Jornadas de Cultura Grecolatina del Sur \& III Jornadas Internacionales de Estudios Clásicos y Medievales "Palimpsestos», Bahía Blanca, Argentina. Sus autores han contado con financiación del Ministerio de Economía y Competitividad del Gobierno de España (Proyecto de Investigación FFI2014-52808-C2-2-P) y del Consejo Nacional de Investigaciones Científicas y Técnicas (Conicet, Argentina). Agradecemos las indicaciones, especialmente bibliográficas, de los revisores anónimos.

Copyright: (C) 2018 CSIC. Este es un artículo de acceso abierto distribuido bajo los términos de la licencia de uso y distribución Creative Commons Reconocimiento 4.0 Internacional (CC BY 4.0). 
diferencias notables en el caso de los «grados del diminutivo». El presente artículo constituye la primera historia completa de la doctrina de los grados del diminutivo en la gramática latina.

Palabras clave: gramáticos latinos; diminutivo; comparativo; derivación. the treatment of «diminutive degrees». This paper is the first complete history of the doctrine of diminutive degrees in Latin grammar.

Key-words: Latin grammarians; diminutive; comparative; derivation.

Cómo citar este artículo / Citation: Burghini, Julia y Uría, Javier 2018: «Los grados del diminutivo: una doctrina confusa en la gramática latina antigua», Emerita 86 (2), pp. 327-348.

\section{Introducción}

Los sufijos -*ko- y -*lo- fueron muy productivos en la lengua latina ${ }^{1}$, en especial en la formación de diminutivos, dado que, mediante secuencias sufijales (por ejemplo, -culus $>-k o-+-l o-)^{2}$, variantes contextuales ${ }^{3}$, combinaciones y reduplicaciones, dieron lugar a múltiples composiciones (-ulus, -ellus, -illus, -culus, -cellus, -ellulus, -cellulus, etc., cf. Leumann 1977, §282,

${ }^{1}$ El sufijo -*lo- se utilizó para formar algunos sustantivos y adjetivos derivados de verbos (tumulus $<$ tumeo; pendulus < pendeo) y para los diminutivos de adjetivos y sustantivos (regulus < rex); mientras que -*ko- (acompañado en general de $\mathrm{i} / \overline{1}$, aparece como -icus $/ \bar{i} c u s$ ) forma adjetivos, como ciuicus (< ciuis), cf. Baldi 2002, pp. 304-307. Dada la ingente bibliografía sobre el diminutivo y los grados de comparación, y habida cuenta de los limitados objetivos de este artículo, las referencias bibliográficas son necesariamente selectivas en lo que se refiere a estudios de tipo general. Los citados a los largo del artículo pueden completarse con las clásicas obras de Hanssen 1951 y Hakamies 1951, y actualizarse con el reciente trabajo de López Gregoris 2016, con abundante bibliografía).

${ }^{2}$ La recursividad sufijal en la formación de diminutivos es muy frecuente en las lenguas; véase al respecto, por ejemplo, Dressler et al. 2015, pp. 266-267, referido al lenguaje infantil.

${ }^{3}$ Por ejemplo, -ellus es resultado fonético regular del sufijo - *lo- añadido a temas en $n$ - o -r. Así: *puer-lo-s > puellus. De ahí puella, en origen, un diminutivo, cf. Prisc., Gramm. II 110.9-19: quae uero geminant l ante us uel a uel um, in ulus ula ulum faciunt iterum diminutiua, ut homullus homullulus, pauxillus pauxillulus, pauxilla pauxillula, pauxillum pauxillulum. feminina quoque in na desinentia, siue habeant ante $n$ aliam consonantem siue non, geminant in diminutiuis $l$ ante a, ut catena catella, asina asella, gemina gemella, columna columnella. excipitur rana ranunculus. eandem formam in la quoque desinentia uel ra seruant, ut fabula fabella, tabula tabella, libra libella, capra capella, umbra umbella, sacer sacra sacrum, sacellus sacella sacellum. puer, puera antiqui, ex quo puella; tener tenera tenerum, tenellus tenella tenellum, miser misera miserum, misellus misella misellum. A partir de estos casos se hace un reanálisis por el cual surge un sufijo -ellus/-ella, por ejemplo, en agellus < *agro-los, cf. Baldi 2002, p. 305. 
para el origen y la historia de los diferentes sufijos y su combinación con diferentes clases de palabras y tipos flexivos). Gracias a ellas se puede obtener un aumento de la función diminutiva (Haverling 2011, p. 227), algo que no escapó a la atención de los gramáticos latinos, que, desde Varrón a Prisciano, comentaron esta riqueza, con frecuencia poniéndola en relación con los grados de comparación ${ }^{4}$. En esos comentarios se centrará el presente artículo, que deja a un lado, por tanto, la espinosa cuestión del origen de los sufijos diminutivos y su relación con otras formaciones ${ }^{5}$.

Las alusiones que Varrón en su De lingua Latina hace a los grados de comparación y a los diminutivos son tratadas sistemáticamente por Manfredini 2007, quien, sin embargo, prescinde de seguir la recepción de esa doctrina en los gramáticos tardíos. Por su parte, el ya citado Haverling 2011 sí recoge los testimonios tardíos, pero no profundiza en su análisis, que cae fuera del objetivo de su contribución. Por ello, el presente artículo constituye la primera historia completa de la doctrina de los grados del diminutivo en la gramática latina antigua, incluyendo la inexcusable referencia a los precedentes griegos.

En las artes grammaticae el tratamiento de los diminutivos tiene, en general, un status mixto: con algunas variantes doctrinales que inmediatamente veremos, desde un punto de vista semántico se destaca su cercanía a los grados de comparación (son contrapunto de estos en tanto representan la disminución de la esencia de un nombre), y desde un punto de vista formal se clasifican como nombres derivados, dado que se derivan de nombres primitivos (Fournier 2013, p. 120).

${ }^{4}$ Cf. ThLL VI 2.2161.38-40 sobre el uso de gradus «ad exemplum comparationis de [gradi]bus deminutiuorum, frequentatiuorum, numeralium, eorumque uerborum quae sequuntur analogiam».

${ }^{5}$ La relación de derivados en -*lo- diminutivos y no diminutivos fue ya objeto de una monografía de Zucchelli 1970, quien dedica también un artículo (Zucchelli 1969) al origen del valor diminutivo del sufijo. Recientemente la cuestión ha sido retomada por Haverling 2011, y, en relación con las lenguas técnicas, por López Gregoris 2005. Los gramáticos latinos tipifican como quasi diminutiua o sono diminutiua palabras del tipo macula fabula, cf. Don., Gramm. 615.5-6 H.; Diom., Gramm. I 326.25-26; Consent., Gramm. V 340.26-29; Pomp., Gramm. V 145.7-10, 220.33-35, 221.1-4, Serv., Gramm. IV 429.23-25. Los gramáticos antiguos son citados por las colecciones de Grammatici Latini y Grammatici Graeci, salvo los casos de Donato (citado por Holtz 1981), Carisio (Barwick 1964), Dosíteo (Bonnet 2005), Sergio (Stock 2005) y Dionisio Tracio (Lallot 1998, en este caso acompañado de página y línea de Uhlig). Las citas del libro VIII de Varrón pertenecen a Dahlmann 1940 y las del IX a Kent 1938. 
Esa doble relación se observa, de hecho, en la posición que, en la mayor parte de las artes grammaticae ${ }^{6}$, ocupan los diminutivos dentro de las species nominum; y es que o bien se sitúan, como en Carisio y Dosíteo, en el tercer puesto entre los nombres derivados ${ }^{7}$, o bien siguen, como en Prisciano y Dionisio Tracio, a comparativo y superlativo ${ }^{8}$. Cuando ocupan una posición diferente, siempre parece deberse a reelaboraciones hechas con diversas finalidades: así, parece claro que el que comparativo y superlativo se releguen en muchos casos al final de la lista se debe al amplio excurso que al respecto suelen hacer los gramáticos, un excurso que, situado en el medio, daría lugar a una ruptura demasiado severa de la enumeración.

No obstante, aunque el parangón con el derivativo y el comparativo es hecho de forma más o menos explícita por la mayor parte de los gramáticos, existen diferencias de detalle, seguramente debidas a la diferente comprensión que unos y otros hicieron de sus fuentes. En el caso de los grados del diminutivo, las diferencias llegan a ser notables, como veremos.

\section{Derivativo y diminutivo}

Desde un punto de vista moderno no sorprende en absoluto la relación de nombres derivados y diminutivos, ya que estos se caracterizan precisamente por un sufijo asociado a un sentido de minoración, disminución o, secundariamente, afecto o desprecio ${ }^{9}$. Suponen, pues, un tipo específico de derivado

${ }^{6}$ Aunque más abajo nos ocuparemos específicamente de ello, cabe adelantar que en el libro VIII del De lingua Latina de Varrón los diminutivos (Varro, Ling. VIII 79) son tratados después del comparativo (Ling. VIII 75-78) e inmediatamente antes del derivativo (Ling. VIII 80-84).

${ }^{7}$ Siguiendo a patronímicos y posesivos, cuya prioridad en la lista se debe al hecho de ser derivados de nombres propios. De hecho, en Donato, que separa, en parte, las species appellatiuorum de las de los nombres propios, el diminutivo (monticulus scholasticulus) sigue inmediatamente al genérico derivativo (montanus scholasticus).

${ }^{8}$ Un listado de las diferentes species nominum en Dionisio Tracio, Diomedes, Carisio, Anonymus Bobiensis, Dosíteo, Donato, Sergio, Probo, Consencio y Prisciano puede encontrarse en Uhlig 1881, pp. 28-29 (que debe manejarse con cautela, pues presenta algunos errores en los números con que representa las diferentes clases).

${ }^{9}$ Por comodidad, y conscientes de la simplificación que supone, agrupamos bajo las etiquetas de «afecto» y «desprecio» diferentes valores pragmáticos (connotativos) que el diminutivo adquiere en el uso, pero que apenas afectan a la argumentación de este artículo. Esos valores secundarios son tratados de una u otra forma en la bibliografía: Hanssen 1951, 
nominal, y como tal aparecen en las clasificaciones que leemos en Dionisio Tracio (D.T. 12.12-13 [25.6-7 Uhlig]), Diomedes (Gramm. I 323.20-23) o Prisciano (Gramm. II 60.1-5). Sin embargo, existe una tradición diferente en la que el diminutivo adquiere una relevancia especial hasta el punto de situarse al mismo nivel que el derivativo, en lugar de como un subtipo de este. Esta tradición está claramente representada en un capítulo de los instituta artium de Probo (Gramm. IV 73.33-74, 16), en el que se señalan tres ordines nominum: positio ('mons'), deriuatio ('montius, montanus'), deminutio ('monticulus'). Esos tres ordines -se nos dice- son posibilidades de los nombres, que, sin embargo, al igual que los grados de comparación (sic uti et in gradibus conlationis), no tienen por qué hallar expresión en todos los nombres: así, por ejemplo, pater tiene derivados (patrius y paternus), pero no diminutivo, mientras que de canis no se conoce derivado, pero sí diminutivo (catulus).

Que esta tradición paralela (¿genuinamente latina?) debió tener un cierto arraigo e influencia lo muestra el hecho de que en la exposición del propio Donato (Don., Gramm. 615.1-4 H.) sobre las species appellatiuorum, tras el par corporalia-incorporalia encontramos el trío primae positionis ('mons schola') - deriuatiua ('montanus scholasticus') - deminutiua ('monticulus scholasticulus').

A la vista de ello, cabe plantearse si no hay en realidad la misma serie en el siguiente pasaje de Carisio:

Char., Gramm. 196.10-27 B. sunt quaedam etiam nomina quae sicut nata sunt efferuntur, ut mons schola: sunt quaedam deriuatiua quae ab aliqua persona propter coniunctionem generis declinantur, ut Pelides Aeacides ... quaedam possessiua, ut Peleius Aeacius ... sunt quaedam diminutiua quae in absolutis nominibus adiecta in nouissima parte aut littera aut syllaba capiunt deminutionem sine ulla comparatione, ut mons monticulus [montanus montaniculus $N]^{10}$, scholasticus scholasticulus, paruus paruulus, adulescens adulescentulus.

por ejemplo, habla de valor peyorativo y usos afectivos; de forma más sistemática, la distinción y relación de los valores denotativos y connotativos se explica en López Gregoris 2016.

${ }_{10}$ A diferencia de Barwick, Keil había preservado la lectura del Neapolitanus (montanus montaniculus), ciertamente más congruente con scholasticus scholasticulus, y que, a diferencia de mons monticulus, no entra en conflicto con el anterior absolutis nominibus ni con el resto de adjetivos que completan la lista de ejemplos. 
A diferencia de la de Donato, la serie carisiana introduce antes del diminutivo las clases de derivativos típicos del nombre propio: los patronímicos (Pelides, Aeacides) y los posesivos (Peleius, Aeacius), que habrían sustituido a los derivados de apelativos (montanus, scholasticus) que esperaríamos para una serie iniciada con los primitivos mons y schola ${ }^{11}$; esa secuencia sí aparece, en cambio, en Diomedes y Dosíteo (este último da en este punto el mismo texto que el Anonymus Bobiensis):

Diom., Gramm. I 323.17-20 sunt quaedam principalia, quae Graeci prototypa dicunt, ut fons mons uilla schola hortus. ex his nascuntur deriuatiua, quae apud Graecos paragoga dicuntur, ut fontanus montanus uillaticus scholasticus horticus.

Dos., Gramm. 21.1-4 B. sunt quaedam primae positionis ut 'mons' 'fons' 'Peleus' 'Aeacus'; quaedam deriuatiua ut 'montanus' 'fontanus' 'Pelides'

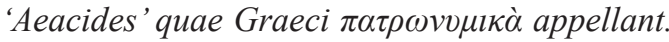

Sin embargo, cuando llegan a la ejemplificación de los diminutivos, tanto Diomedes como Dosíteo y el Anonymus Bobiensis se limitan a ofrecer paruus paruulus y adulescens adulescentulus, con lo que su testimonio no sirve para reconstruir el de la fuente común del grupo de Carisio ${ }^{12}$, que incluye, junto a este autor, a Dosíteo y al Anonymus Bobiensis. Todo apunta, no obstante, a que esa fuente había ilustrado diminutivo y derivativo exclusivamente con ejemplos referidos a personas (nótese ab aliqua persona en el texto de Carisio), y a que contemplaba el diminutivo como una derivación secundaria respecto al derivativo.

\section{Comparativo y diminutivo}

En su forma prototípica (altior, altius), los comparativos latinos son adjetivos modificados por un sufijo que señala, respecto a la propiedad expresada

${ }^{11}$ Hasta donde conocemos, está por estudiar el papel que la distinción entre nombres propios y apelativos tiene en toda la organización de las species nominum; solo Diomedes (parcialmente) $\mathrm{y}$, al final de la tradición, Prisciano hacen un esfuerzo por distinguir las species que son exclusivas de propios (Prisc., Gramm. II 57.12-13) o de apelativos (Prisc., Gramm. II 59.20-24), o compartidas por ambos (Prisc., Gramm. II 59.9-19). Vaahtera 1998, p. 62, hace notar que la «confusion between proper names and appellatives is common in Roman grammarians».

${ }_{12}$ Sobre la composición del «grupo de Carisio», véase Barwick 1922, pp. 7-10, y Holtz 1981, p. 81 . 
por el adjetivo, que algo o alguien la posee en un grado mayor; por ello, con mayor frecuencia forman parte de estructuras comparativas en las que interviene, además del marcador de grado o sufijo comparativo propiamente dicho («degree/parameter marker»), el llamado primer término de la comparación («first term of the comparison», «comparee or topic of comparison»), el adjetivo al que se añade el sufijo («parameter or predicate»), una partícula comparativa («standard marker or pivot») y un segunto término de la comparación («second term of the comparison», «standard of comparison») ${ }^{13}$. Como en español, también en latín la comparación de grado se limita a adjetivos y adverbios, de tal manera que incluso cuando se fuerza su aplicación a sustantivos, estos son, de hecho, convertidos en adjetivos ${ }^{14}$. No en vano los gramáticos latinos, que consideraban la comparación un «accidente» del nombre, la utilizaban para dirimir el estatuto de palabras ambiguas como nombres/participios del tipo sapiens, prudens ${ }^{15}$.

Por el contrario, el diminutivo, aunque comparte con el comparativo la formación sufijal, se forma preferentemente sobre sustantivos (secundariamente también sobre adjetivos) y no participa de ninguna construcción sintáctica peculiar. Por ello, la sufijación diminutiva se considera más un procedimiento derivativo que flexivo, a diferencia de la sufijación comparativa ${ }^{16}$.

Esa distinción, sin embargo, no es patente en la gramática antigua. En efecto, ni siquiera en Varrón, que discute en detalle la diferencia entre flexión (declinatio naturalis) y derivación (declinatio uoluntaria), está claro a cuál de los dos procesos se asignan los comparativos y diminutivos, cuando se mencionan juntos en el libro VIII:

Varro, Ling. VIII 52 de nominatibus quae accedunt proxime ad infinitam naturam articulorum atque appellantur uocabula, ut homo equus, eorum de-

${ }^{13}$ La terminología inglesa a la que adaptamos la española está tomada de Tarriño 2011, pp. 375-376 y de Cuzzolin y Lehmann 2004, p. 1212.

${ }^{14}$ Como se puede observar comparando Los alemanes son más hombres que los españoles (comparación de grado; hombres [adj.] = viriles) y En Alemania hay más hombres que en España (comparación de cantidad hombres [sust.] = seres humanos de sexo masculino). Algo similar se observa en la gradación con el adverbio muy: Son muy hombres frente a Son muchos hombres.

${ }^{15}$ Cf., por ejemplo, Sacerd., Gramm. VI 444.2-5, Cled., Gramm. V 72.10-14 y Char., Gramm. 238.9-18.

${ }^{16}$ No obstante, los especialistas discuten si es posible trazar siempre una frontera entre flexión y derivación. Una síntesis de las distintas posturas y criterios puede leerse en Booij 2000. 
clinationum genera sunt quattuor, unum nominandi ut ab equo equile, alterum casuale ut ab equo equum, tertium augendi ut ab albo albius, quartum minuendi ut cista cistula.

Para Flobert 1989, pp. 746-747, Varrón, a imitación de los griegos ${ }^{17}$, privilegia aquí ${ }^{18}$ comparativos y diminutivos por buscar una simetría, aunque -apunta el autor francés- los comparativos están gramaticalizados y los diminutivos no. Por su parte, Iso 1997-1998, pp. 64-65 hace notar que diminutivos y comparativos -que en Ling. VIII 52 aparecen coordinados para ejemplificar los tipos de declinatio de los nombres comunes- en un esquema anterior (Ling. VIII 14) constituían subdivisiones de las relaciones lógico-semánticas que se establecen entre nombres derivados y sus primitivos (concretamente, de las que expresan un cambio del mismo objeto, que se diferencian de aquellas derivaciones que suponen cambio de objeto). En cambio, no se deduce necesariamente de Ling. VIII 52 que la derivación ab equo equile era, para Varrón, uoluntaria mientras que las otras tres eran ejemplos de declinatio naturalis, es decir, de flexión ${ }^{19}$ : esa distinción no parece estar en la mente de Varrón cuando escribe el párrafo $52^{20}$.

Es difícil asegurar cuál sería la postura de Varrón respecto a diminutivos y comparativos en relación con los derivativos, dado que estos últimos suscitaron una gran controversia desde el final de la República, testimoniada tanto en el De lingua Latina como en el De analogia de Julio César ${ }^{21}$, y, más tarde, en Plinio. Testimonios posteriores hacen pensar que no existió un acuerdo al respecto: así, mientras Pompeyo parece admitir una cierta regula-

17 Véase también Dahlmann 1940, pp. 136-137.

18 No en Ling. VIII 14, como indica por error Flobert 1989, p. 746.

19 Es la interpretación del pasaje por Manfredini 2007, p. 205: «El primer tipo morfológico es el que corresponde a la declinatio uoluntaria, es decir a la derivación por sufijación; el segundo, el caso, pertenece a la naturalis, y los otros dos, en torno a los cuales giran nuestras propios comentarios, como no participan de la impositio, que es el acto de poner nombre a las cosas a través del lenguaje, acción congénita de la declinatio uoluntaria, entroncan en el tramado de flexiones communi consensu articuladas por los hablantes de la lengua, condición esencial de la declinatio naturalis.»

${ }^{20}$ Posición opuesta a la de Manfredini la expresa Viti 2014, p. 287 n. 6, para quien Varón ofrece de forma explícita («explicitly») un análisis derivacional de la comparación, al considerar las formas comparativas igual que las diminutivas.

${ }^{21}$ Véase Garcea 2012, pp. 162-167. 
ridad en los diminutivos ${ }^{22}$, en Carisio se asimila la irregularidad de derivativos y diminutivos ${ }^{23}$, en un pasaje en el que no está en cuestión, como en Varrón (Ling. VIII 7924; cf. IX 74), la irregularidad entendida en términos de «defectividad», es decir, la que, al igual que los comparativos, tienen los diminutivos al formar, ya cero (cadus), ya uno (lectus lectulus), ya dos «grados» de disminución (cista cistula cistella); antes bien, lo que el pasaje de Carisio pone de relieve es la variedad sufijal de los diminutivos, que hace imposible predecir de manera analógica (por la ratio) qué forma adoptará el diminutivo de una palabra.

A diferencia de la anterior, esta otra vertiente de la irregularidad de los diminutivos no es, en latín, trasladable a los comparativos (que se forman casi sin excepción con el sufijo -ior, -ius), y solo de forma limitada a los superlativos (para los que el sufijo -issimus es de uso mayoritario en comparación con la reducida extensión de -mus y -errimus). Hay que hacer notar al respecto que en griego la situación que describen los gramáticos es más uniforme, pues tanto para comparativos y superlativos como para diminutivos se señala la variedad de formaciones. Así, en Dionisio Tracio a propósito del comparativo se indica expresamente que sus $\tau u ́ \pi o l$ son tres:

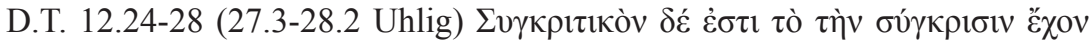

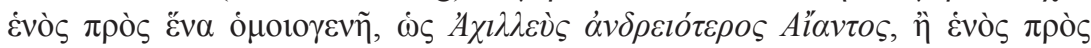

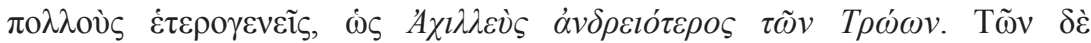

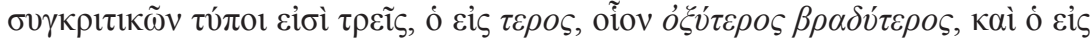

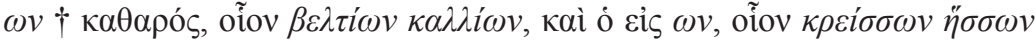

${ }^{22}$ Véase Pomp., Gramm. V 144.7-8 et de principalibus et de diminutiuis est ratio; de deriuatiuis nulla ratio est, con el oportuno comentario de Garcea 2012, p. 163: «the grammarian Pompeius states that although diminutives are formed regularly, or at least use a regular series of suffixes, derivatives do not appear to follow any identifiable norms».

${ }^{23}$ Char., Gramm. 119.12-120.1 B. deriuationis uero tanta est inaequalitas ut conprehendi non possit ... de qua quaestione a Velio Longo libellus scriptus est. item deminutionis inaequalitas dura est ut iunenis iunenculus, canis catulus, puluis puluiculus, uinum uinulum, talus taxillus, panis pastillus, homo homunculus et homuncio, piscis pisciculus et pisculus.

${ }^{24}$ La edición de Goetz-Schoell 1910 aduce el pasaje de Carisio como paralelo del de Varrón, mientras que el comentario de Dahlmann 1940, p. 180, distingue claramente la «Uneinheitichkeit der Zahl der Diminutivgrade» que pone de relieve Varrón, de la «Verschiedenartigkeit der Bildungen» que señala Carisio. 
Comparativo es el que presenta la comparación de uno respecto a otro del mismo género, como "Aquiles es más valiente que Áyax», o de uno respecto a muchos de distinto género, como «Aquiles es más valiente que los troyanos». Los tipos de comparativos son tres: en - $\tau \varepsilon \rho \circ \varsigma$, como ỏ $\xi u ́ \tau \varepsilon \rho \circ \varsigma$ ('más rápido'), $\beta \rho \alpha \delta$ $\tau \varepsilon \rho o \varsigma$ ('más lento'); en - $\omega v$ puro, como $\beta \varepsilon \lambda \tau i ́ \omega v$ ('me-

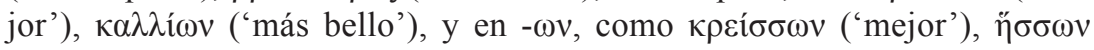
$(\text { 'peor') })^{25}$.

asimismo, del superlativo se describen dos $\tau u ́ \pi o v:$

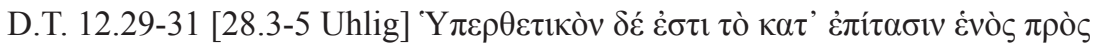

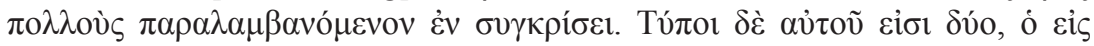

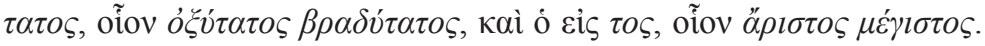

Superlativo es el empleado en una comparación para la intensificación de uno

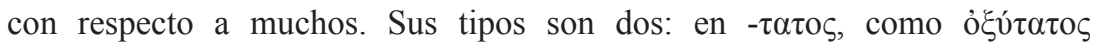

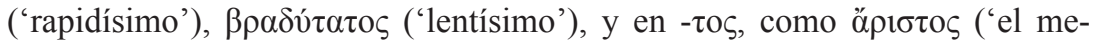
jor’), $\mu \varepsilon ́ \gamma \imath \sigma \tau o \varsigma$ ('el más grande’).

Del diminutivo, solo se menciona la definición semántica y nada dice de sus $\tau u ́ \pi o r$, aunque de los ejemplos se deduce que el gramático los contempla, dado que presenta ejemplos con los sufijos «regulares» del diminutivo en griego (-í $\sigma \kappa \circ \varsigma$ e -10v) y con un tercero, $-\alpha \xi$, que se encuentra más frecuentemente asociado a un valor peyorativo (especialmente aplicado a personas) que a uno diminutivo ${ }^{26}$ :

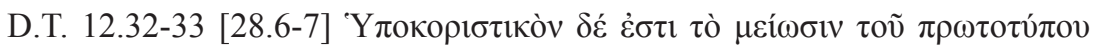

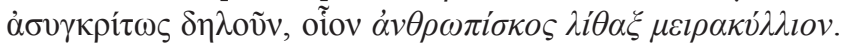

Diminutivo es el que expresa una disminución del primitivo sin comparación, como 'hombrecito', 'piedrecita', 'jovencito'.

${ }^{25}$ La traducción de los pasajes de Dionisio Tracio es de Bécares Botas 2002.

${ }^{26}$ Véase al respecto Peppler 1902, p. 42, que distingue un sufijo diminutivo $-\alpha \xi$ ( $\beta \tilde{\omega} \mu \alpha \xi$, $\lambda i \theta \alpha \xi)$ de uno peyorativo $-\bar{\alpha} \xi$, atribuyendo a la creatividad de la comedia y de la lengua popular la acuñación de palabras como $\pi \lambda$ ov́ $\tau \bar{\alpha} \xi, \theta \alpha \lambda \alpha \dot{\alpha} \mu \bar{\alpha} \xi$. Chantraine 1979, p. 381, por su parte, apunta que el sufijo en $-\bar{\alpha} \kappa$ ha sido utilizado para formar derivados secundarios de sentido peyorativo, y que probablemente sea un préstamo de la comedia siciliana al jónico-ático (así se explica $\bar{\alpha}$ por $\eta$ ). 
Los tipos formales del diminutivo griego, reducidos por Dionisio Tracio a tres ${ }^{27}$, serán sensiblemente ampliados por comentaristas de su manual. Por ejemplo, en Sch. D.T. 227.7-24 (cf. 376.6-24 y 539.4-14) se distinguen seis sufijos masculinos $(-\omega v,-i \omega v,-\alpha \xi,-\alpha \varsigma,-\sigma \kappa o \zeta,-\lambda \circ \varsigma)$, tres femeninos $(-\omega,-1 \zeta$, $-\sigma \kappa \eta)$, y un solo neutro en -ov, del que se reconocen seis variantes (diez en 376.17-24) según la sílaba precedente: -vi-ov, - $\delta i ́-o v,-\rho \imath-o v,-\delta \rho \imath-o v,-\lambda \imath-o v$ y - $\varphi$-ov. Sin embargo, pese a la variedad y cantidad de sufijos reconocidos, no se encuentra en los gramáticos griegos ${ }^{28}$ una doctrina paralela a la que entre los romanos se desarrolló sobre los grados del diminutivo, y no por falta de ejemplos sobre los que construir una teoría similar ${ }^{29}$. A este respecto, es interesante recordar que Aristóteles, en su Retórica (1405b), aconseja la moderación en el uso de diminutivos, refiriéndose con ello a la innecesaria acumulación de sufijos, como la que había utilizado Aristófanes con intención satírica. Por consiguiente, es plausible pensar que la recursividad sufijal en la formación de diminutivos era vista como algo ajeno a la lengua formal, y más propio de la lengua popular y de géneros extraños al canon ${ }^{30}$.

\section{Los grados del diminutivo}

La posibilidad de que, al igual que el comparativo, también el diminutivo desarrolle dos grados es -ya se ha dicho- contemplada en primer lugar por

${ }^{27}$ Parece evidente que la escasa representación de sufijos tiene que ver con los textos de referencia de la gramática griega, esencialmente Homero, cuya «evitación» de los diminutivos ha sido comentada desde la Antigüedad (por ejemplo, en Sch. D.T. 226.12-21), y, en menor medida, los líricos e historiadores.

${ }^{28}$ Esta ausencia es señalada por Dahlmann 1940, p. 180: «So weit wir sehen, hat die griechische Grammatik das nicht gehabt; die Dionysiusscholien unterscheiden lediglich sechs verschiedene, aber nicht graduell abgestufte $\tau$ tó männlicher, drei weiblicher und eins neutraler vंлокорıблєка́...».

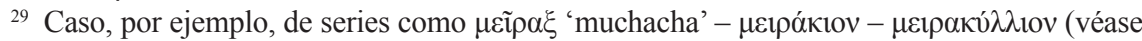
Leumann, 1953). La acumulación de sufijos diminutivos en la lengua griega es típica de la comedia (Peppler 1902, pp. 11-13; Chantraine 1979, p. 76). Por ello, no resulta extraño que nombres como Phronesium, Philocomasium, etc., tan típicos de la comedia latina, procedan de diminutivos griegos en -10v, aunque es problemático saber si el espectador romano percibía ese valor.

30 Entiéndase esta apreciación en relación con la observación de Aristóteles y referida a los verdaderos diminutivos, sin tener en cuenta la abundacia de formaciones sufijales «diminutivas» en textos técnicos, por ejemplo (respecto a esto último véase López Gregoris 2005, con bibliografía, a la que puede añadirse Adams 1995, pp. 543-551). 
Varrón, que la ilustra con el ejemplo cista - cistula - cistella en Ling. VIII 79 (donde ya aparece la palabra gradus ${ }^{31}$ ), ejemplo al que añadirá canis catulus - catellus en Ling. IX 74, un pasaje en el que, además, reconoce -utilizando de nuevo la palabra gradus - que lo normal es establecer no tres, sino solamente dos dimensiones de disminución (lectus - lectulus, arca - arcula). Es incontestable (Dahlmann 1940, p. 180) que en Varrón la doctrina de los grados del diminutivo está inspirada por el inmediatamente precedente (Ling. VIII 75-78) tratamiento del comparativo, con el que ya se había agrupado en Ling. VIII 52, al constatar los declinationum genera de los sustantivos (tertium augendi, ut ab albo albius, quartum minuendi, ut a cista cistula).

El contexto en que Varrón esboza la doctrina de los grados es el de la crítica anomalista a la aplicabilidad de la analogía a los nombres. Es ese contexto el que facilita la construcción ideal de dos grados de disminución (paralelos a los dos grados de aumento que implican comparativo y superlativo), lo que da lugar a la abstracción de una estructura trimembre (a partir de ejemplos como cista - cistula - cistella) de la que, como antes se hace con los comparativos (Ling. VIII 76, IX 72), se señala la defectividad, argumentando, por ejemplo, la inexistencia de un nigricolus que hubiera servido de primer grado de disminución de niger (nigellus sería, a la vista de cistella, el segundo grado $)^{32}$.

Otro aspecto interesante del planteamiento de Varrón es que asume la motivación semántica (magnitudo, gradus) de los grados de disminución, como se deduce de sus palabras en Ling. IX 74 (Ad haec respondeo huiuscemodi uocabulis analogias esse, ut dixi, ubi magnitudo animaduertenda sit in unoquoque gradu eaque sit in usu communi, ut est cista cistula cistella et canis catulus catellus, quod in pecoris usu non est), que implican el reconocimiento de una extensión parcial de la regularidad analógica, limitada a los casos en que la realidad permita la distinción de esos tres grados de tamaño (cista - cistula - cistella), algo que, según se reconoce de inmediato, no es precisamente lo más común, ya que la distinción de dos grados de tamaño

${ }^{31}$ Seguramente en Varrón no tiene aún el sentido técnico que adquirirá más adelante. Por el ThLL (VI 2.2161.37-62; cf. Schad 2007, p. 190) sabemos que gradus es utilizada por los gramáticos, como se ha apuntado más arriba, también para señalar diferentes dimensiones de, por ejemplo, los verbos derivativos.

${ }^{32}$ Consideraciones similares se hacen para auicella y capitellum, inexistentes (en época de Varrón) segundos grados que corresponderían al primer grado auicula, capitulum. 
(lectus - lectulus) se presenta como la más frecuente. Da la impresión, pues, de que solo un pertinaz empeño por asimilar los grados de disminución a los de comparación es lo que conduce a señalar como regular (analógico) lo que resulta menos común en la lengua. En Ling. VIII 79 había señalado la serie triple como una posibilidad (cum possint esse terna) a partir de la cual se indicaban como irregularidades la falta del primer (macer - *macricolus macellus) o el segundo grado (auis - auicula - *aucella) de disminución, con lo que se acababa concluyendo que en realidad este tipo de nombres se guiaba más por el uso que por la analogía (in hoc genere uocabulorum quoniam multa desunt, dicendum est non esse in eo potius sequendam quam consuetudinem rationem).

Esa motivación semántica sobre cuya existencia Varrón no parece dudar es precisamente la que genera cierto desacuerdo -rayano en la contradicciónen algunos de los desarrollos que los gramáticos tardíos hacen de la doctrina de los grados. De ello nos ocuparemos a continuación.

Comencemos advirtiendo que no todas las artes grammaticae se ocupan de la doctrina de los grados, sino que esta es enunciada solamente en Donato y sus comentaristas, en Consencio y en Diomedes, con ecos más tardíos, ya fuera de los manuales, en Macrobio y en los comentarios de Donato a Terencio. No está representada, en cambio, en el denominado grupo de Carisio.

El enunciado básico de la doctrina puede ejemplificarse con el texto de Donato, que tiene paralelos en Consencio y Diomedes, con ligeras variantes y un cierto desarrollo (significativo en Diomedes):

Don., Gramm. 615.3-6 H. alia sunt primae positionis, ut mons, schola; alia deriuatiua, ut montanus, scholasticus; alia diminutiua, ut monticulus, scholasticulus. diminutiuorum tres sunt gradus; quorum forma quam magis minuitur, crescit saepe numerus syllabarum. sunt etiam quasi diminutiua, quorum origo non cernitur, ut fabula, macula, tabula, uinculum.

Consent., Gramm. V 340.21-29 sunt etiam diminutiua ut monticulus scholasticulus. diminutiua autem non solum a positiuo, sed etiam a conparatiuo gradu interdum ueniunt. nam ut grandis grandiculus facit, sic grandior grandiusculus. diminutiuorum autem tres sunt gradus, quorum forma quam magis minuitur, saepe crescit numerus syllabarum. sed in diminutiuo gradu hoc animaduertere debemus, ne forte fallamur, quod sunt quaedam nomina quasi diminutiua, quae diminutiua non sunt, quoniam prima positio eorum non cernitur, ut uinculum macula tabula fabula. 
Diom., Gramm. I 325.25-326.10 diminutiua sunt quae in diminutione absolutorum nominum fiunt sine ulla conparatione, ut paruus paruulus, adulescens adulescentulus. horum autem tres sunt gradus. quorum forma quamuis magis $^{33}$ minuitur, crescit saepe numerus syllabarum. ... ${ }^{34}$ apud antiquos aliae diminutiones reperiuntur. descendebant enim ad tertiam usque formam, tam quam arca arcula arcella arcellula, catinus catinulus catellulus, oculus ocellus ocellulus. ex secunda diminutione quaedam sunt in consuetudine, pauca ex tertia, ut ocellulus et catellulus. apud nos diminutionis hoc genus seruatur quod est primae positionis, id est prima diminutio.

Los tres textos (con la salvedad señalada para Diomedes en n. 3) parecen resaltar la paradoja que supone que una disminución formal conlleve aumento del número de sílabas; da la impresión de que subyace la presunción de que el acrecentamiento de la masa fónica de la palabra debería ir aparejada (como de hecho ocurre normalmente en la serie positivo - comparativo - superlativo) no a una disminución sino a un aumento. El carácter formal de la observación está garantizado por el uso de la palabra forma, que se refiere únicamente a la forma física, la apariencia formal (i. e., al significante ${ }^{35}$ ), y de ninguna manera puede confundirse con el significado o sensus ${ }^{36}$. De he-

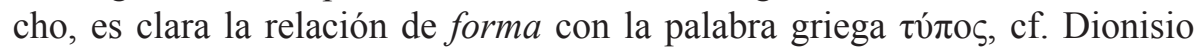

${ }^{33}$ Es quamuis magis la lectura del manuscrito A, mientras que B presenta quam magis y $\mathrm{M}$ solo quamuis. Aunque quamuis no da un sentido muy claro, tiene la ventaja de ser lectio difficilior y podría cambiar completamente el sentido del pasaje: Diomedes alteró el texto que copiaba para darle un mejor sentido, y así salvar la aparente contradicción de que una forma disminuya y a la vez crezca en número de sílabas: una posible lectura alternativa sería ... quam ui magis minuitur, crescit saepe ... «cuya forma, cuanto más disminuye en significado, crece con frecuencia su número de sílabas». En todo caso, aunque supone cierta dificultad, el texto editado por Keil puede entenderse sin enmiendas: «cuya forma [sc. la de los grados] aunque disminuye (cada vez) más [i. e. aunque va en progresiva disminución], su número de sílabas crece con frecuencia». Si se acepta la propuesta quam ui [s] magis, entonces Diomedes se sitúa en la línea de los que observarán una contradicción entre la disminución del sentido y el aumento de la forma. Dammer 2001, p. 107, al igual que Bölte 1886, p. 13, prefiere la lectura de B, teniendo en cuenta el texto de Donato y de Consencio.

${ }^{34}$ Entre 325.28 y 326.5, Diomedes intenta sistematizar los sufijos diminutivos que corresponden a cada declinación, detalles que no afectan a lo que nos ocupa, por lo que suprimimos esa parte del texto.

${ }^{35}$ En ocasiones en claro contraste con el significado, como señala Schad 2007, p. 176. También hay que destacar la frecuencia con que forma alude a derivados nominales y verbales.

${ }^{36}$ Error que cometió Dezotti 2011 en su versión del pasaje de Donato, en tanto tradujo forma como «idéia». 
Tracio supra, (cf. Bécares Botas 1985, p. 386). En consecuencia, estos textos parecen querer decir que cada uno de los sufijos diminutivos (forma graduum) se disminuyen unos a otros, pero no se comprometen afirmando que ello implica una disminución mayor en el sentido ${ }^{37}$. En otras palabras, es posible que Donato, seguido por Consencio, eluda conscientemente la referencia al contenido (sensus) porque percibe que la disminución semántica no siempre acompaña a la formal: el tercer grado del diminutivo no necesariamente es «más pequeño» que el segundo grado ${ }^{38}$. Como afirma Strodach 1933 , p. 51, n. 67, la distinción entre los sufijos diminutivos -ulo/a-, -ello/a(-illo/a), y -ellulo/a- (-illulo/a-) «was one of emotional nuance rather than one of progressively diminutive meaning $»^{39}$.

Lo que para el texto de Donato estamos simplemente deduciendo del uso de la palabra forma lo encontramos efectivamente argumentado en un texto posterior e independiente, debido a Macrobio, que comparte y hace explícita la idea de Donato en el siguiente pasaje:

Macr., Exc. gramm. V 651.14-18 $8^{40}[$ Frg. Bobiense de uerbo = ad Seuerianum 50.23-27 Passalacqua] De frequentatiua. huius formae uerba non numquam uno gradu, non numquam duobus deriuantur, ut scribo scripto scriptito, cano canto cantito, salio salto saltito. nec tamen est in posterioribus maior quam in prioribus frequentationis expressio, sicut nec in diminutiuis secundus gradus minus priore significat, anus anula anicula.

Por el contrario, resulta llamativo que algunos comentaristas de Donato renuncien al uso de la palabra forma, que sacrifican en beneficio del término

${ }^{37}$ Actualmente, la Nueva gramática de la lengua española de la Real Academia 2009, 9.1g, se limita a mencionar el fenómeno: «A pesar de que los afijos apreciativos son sufijos, es posible encadenar varios morfemas apreciativos con idéntico significado dentro de la misma palabra (...): chiqu-it-ito». Es posible que Donato y sus seguidores consideren la duplicación de los sufijos como un refuerzo diminutivo que no implica necesariamente aminoración semántica.

${ }^{38}$ Haverling 2011, p. 227 da cuenta de eso en Plauto: «L'on peut employer plusieurs formes différentes pour indiquer la même chose. Nous retrouvons ce système quand Plaute emploie cistula, cistella et cistellula pour indiquer la même petite corbeille».

${ }^{39}$ Por ejemplo, el adjetivo frīgidulus tiene «a connotation of pity for Ariadne, abandoned by Theseus» en Catul. LXIV 131, cf. Fruyt 2013, p. 12; Hanssen 1951, pp. 9-11; López Gregoris 2016, pp. 186-187.

${ }^{40}$ Pasaje prácticamente idéntico en Macr., Exc. gramm. V 626.10-15. 
-ciertamente más esperable en el contexto- sensus. Con ello, la paradoja que se establecía únicamente en el plano del significante pasa a ser un conflicto de significante y significado: palabras con más masa fónica designan objetos con menor masa física. Textos que ilustran esta idea, en cierto modo contradictoria -insistimos- con la del texto que comentan, son los de Servio y Pompeyo:

Serv., Gramm. IV 429.21-24 sunt etiam diminutiua, quae sensum minuunt et non semper, sed frequenter syllabis crescunt, ut agnus agnellus. sunt etiam alia sono diminutiua, ut tabula fabula.

Pomp., Gramm. V 143.19-144.7 sed deriuatio nominis deductionem habet sine sensu, diminutio sensum diminuit. hoc interest inter deriuationem et diminutionem, quod deriuatio nomen facit, non aut maius aut minus, sed nomen facit. mons, montanus dictus est. numquid montanus est aut qui maior est aut qui minor est? non, sed nomen habet. e contrario diminutio non solum nomen facit, sed et sensum diminuit, ut puta fons, fonticulus et nomen est et sensus diminutus. haec ipsa diminutio plerumque crescit; non semper, sed plerumque crescit. aliquotiens una diminutio est, aliquotiens duae, aliquotiens tres, quem ad modum potueris inuenire. ut puta agnus principale est: fac diminutiuum, agnellus; aliud, agnicellus; aliud diminutiuum, agnicellulus. potes inuenire in infinitum et multas diminutiones, quae praecipue aptae sunt lyricis. sed scire debes, ait, quoniam haec ipsa diminutio hac arte facienda est, ut, quando sensus minuitur, crescat numerus syllabarum. puta mons una syllaba est: monticellus creuit, quattuor sunt syllabae: monticellulus quinque sunt syllabae. sed quoniam scimus istas res non posse semper contingere, idcirco et ipse moderatus est et ait sic, quando sensus minuitur, crescit saepe numerus syllabarum. non dixit semper. inuenimus secundam et tertiam diminutionem; sensum quidem minuit, in isdem tamen syllabis permanet: quot habuit prima positio a natura, tot habent syllabas et diminutiones, licet sensus minuatur. ergo principalia sunt quae a natura fiunt, deriuatiua quae inde nascuntur, diminutiua quae etiam sensum minuunt.

Servio cambia la fraseología donatiana y afirma que los diminutivos reducen el sentido (sensum minuunt) y no siempre, aunque con frecuencia, aumentan en las sílabas. No menciona de manera explícita que los grados del diminutivo son tres - de hecho, presenta un ejemplo binario, agnus agnellus-, y la mención al aumento de sílabas está relacionada con respecto al grado absoluto (i. e., el nomen primae positionis): los diminutivos reducen el sentido de la palabra primitiva y a veces aumentan el número de sílabas de esta. 
De la misma manera, Pompeyo hace una interpretación claramente alusiva a lo semántico (quando sensus diminuitur), y, por lo tanto, poco fiel a Donato. Tampoco habla de «grados» de los diminutivos, sino de diminutiones, y menciona que en ocasiones se encuentran una segunda y tercera «disminución» (de sus ejemplos se deduce que piensa en casos como monticellus y monticellulus en relación con monticulus, que sería la prima diminutio). Es interesante que destaque el uso donatiano del adverbio saepe en lugar de semper, y que aclare al respecto que, en ocasiones, aunque disminuya el sentido, el diminutivo puede tener el mismo número de sílabas que el primitivo.

Otros comentaristas de Donato permanecen más fieles a su fuente y no efectúan, al menos expresamente, la interpretatio semántica que hemos propuesto para Servio y Pompeyo. Así, las anónimas Explanationes in artem Donati preservan insistentemente la palabra forma de su fuente:

Explan. in Don., Gramm. IV 536.34-37 sunt diminutiua, quae primae positionis per incrementum syllabae minuunt formam, ut mons monticulus, lapis lapillus, caput capitulum, uacca uaccula, ora oscilla. horum nominum quo magis dictione minuuntur formae, eo magis accrescunt syllabae.

Por su parte, en el comentario transmitido bajo el nombre de Sergio ${ }^{41}$, se adopta una fraseología (cum diminuuntur nomina) que no se compromete ni con la interpretación formal ni con la semántica:

Serg., Comm. 8.7-22 Sunt primae positionis, hoc est unde deriuatiua et diminutiua ueniunt, ut schola, nam a schola deriuatiuus 'scholasticus', diminutiuus est 'scholasticulus'. Sciamus autem tres gradus diminutionis esse secundum Donatum, per quos, cum diminuuntur nomina, saepe crescit numerus syllabarum, ut scholasticulus, scholasticellus, scholasticellulus. Apud antiquos uero aliter dicebantur: agnus agnulus, agnellus, agnicellus, agniculus, agnicellulus. Quam rem Donatus uidens, ne in infinitum diminutio foede procederet, uoluit diminutionis tres tantum gradus esse. Saepe autem idcirco dixit augeri numerum syllabarum, quod inueniuntur quaedam nomina, quorum in diminutione non modo non crescit, sed etiam minus habet syllabarum gradus ulterior, ut homo, homuncio, homunculus, homulus. editor.

${ }^{41}$ Conocido también como Pseudo-Casiodoro por la atribución realizada por su primer 
También da cuenta Sergio, al igual que Pompeyo, de la numerosa cantidad de diminutivos que pueden formarse a partir de un primitivo, y por eso justifica el hecho de que Donato establezca que solo hay tres grados para no mutiplicar interminablemente la disminución: ne in infinitum diminutio foede procederet. Es cierto que, aunque los gramáticos suelen indicar normalmente dos grados, o a lo sumo tres, se encuentran en los comentaristas ejemplos de una quarta diminutio, representada por pauxillulum ${ }^{42}$ :

Don., Ter. Phorm. 37 PAVXILLVLVM quartus gradus diminutionis: paulum paululum pauxillum pauxillulum.

Pero la doctrina de los grados del diminutivo parece extinguirse, al menos en la Antigüedad, con los comentarios y comentaristas de Donato. Después del s. V, la monumental obra de Prisciano da de los diminutivos una visión similar a la que conocemos por Dionisio Tracio y sus escolios ${ }^{43}$. Y es que,

${ }^{42}$ Respecto a esta formación véase la explicación de Fruyt 2005, p. 231: «Sur un lexème (adjectif ou adverbe) déréalisant (signifiant 'petit, peu, pauvre, etc.') comme paruus 'petit', l'addition de -ulus produit un renforcement du sème de petitesse et une intensification: paruulus signifie 'tout petit'. La base et le suffixe concordent alors dans leur caractère déréalisant et se renforcent. On arrive ainsi à un résultat qui aurait pu paraître paradoxal au premier abord: un morphème déréalisant signifiant 'un peu' a le même effet qu'un morphème réalisant intensifiant comme le préfixe per-. Dans l'exemple suivant, la désintensification, prenant la réalisation particulière de la petite taille d'une entité discrète, est dénotée par trois morphèmes, qui se renforcent l'un l'autre: les suffixes de diminutif -culus (dans nauiculam) et -ulus (dans paruulam) et le lexème paruus 'petit', en lui-même désintense : nauiculam paruulam (Caes. civ. 3,104,3) 'un tout petit canot'. L'adverbe paululum signifie 'un tout petit peu' (sur paulum 'un peu' + suffixe $-* l o-$ ) avec un renforcement de la valeur déréalisante de la base et un effet d'intensification».

${ }^{43}$ Prisciano se diferencia del resto de los gramáticos en dos aspectos fundamentales: 1) su definición del diminutivo es idéntica a la de la Též

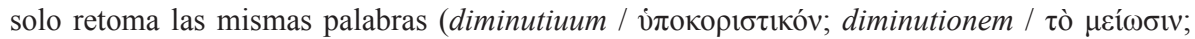

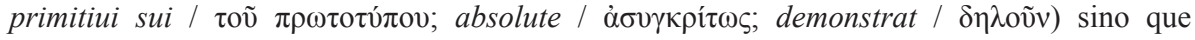

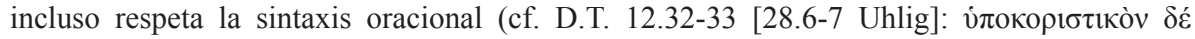

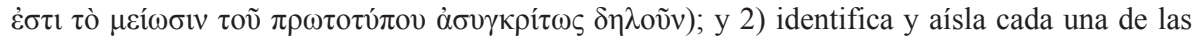

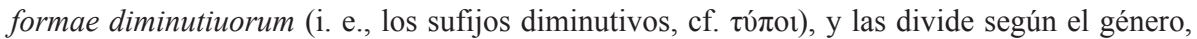

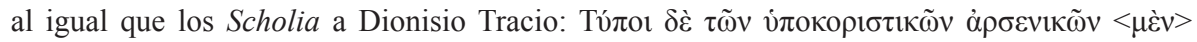

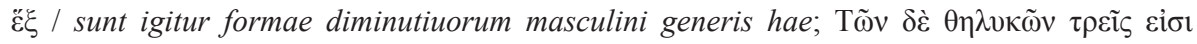

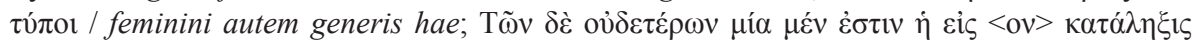
/ neutrorum quoque sunt formae hae, un enfoque que no se encuentra en la gramática latina previa a Prisciano. 
pese a que reconoce y comenta la variedad de sufijos diminutivos, prescinde de conceptualizarlos como grados, con lo que parece verlos más bien como «variantes». Es lo que parece deducirse del siguiente pasaje:

Prisc., Gramm. II 102.1-19 deriuantur igitur pleraque ab appellatiuis, pauca etiam a propriis, et seruant genera primitiuorum plerumque et saepe inueniuntur diminutiuorum diminutiua in diuersas desinentia formas, ut homo, homuncio, homunculus, homullus, homullulus. sunt igitur formae diminutiuorum masculini generis hae: culus, ulus absque c, olus, ellus, xillus, illus absque x, ullus, cio, aster, leus, tulus: culus, ut igniculus; ulus absque c, tantulus; olus, Sergiolus, capreolus; ellus, agellus; illus $x$ antecedente, paxillus, absque x, codicillus; ullus, homullus; cio, homuncio; aster, parasitaster; leus, eculeus, aculeus; tulus, nepotulus. Plautus in milite glorioso: "si te saluum hinc † amittimus Venerium nepotulum». feminini autem generis hae: cula, ula absque c, ola, ella, xilla, illa absque x, ulla, ut anicula, siluula, unciola, capella, maxilla, anguilla, una ulla. neutrorum quoque sunt formae hae: culum, ulum sine c, olum, ellum, illum cum $x$ et sine eo, ullum, ut corpusculum, corculum - Plautus in Casina: «meum corculum, melculum, uerculum», sine c, capitulum, laureolum, lucellum, uillum, uexillum, ullum.

\section{Conclusiones}

Que sepamos, la noción de «grados» del diminutivo no existe en los textos gramaticales griegos conservados, y es posible que ni siquiera llegara a desarrollarse en el ámbito griego, posiblemente porque los textos canónicos en que se fundamentó la gramática alejandrina no frecuentaban el uso del diminutivo. En la gramática latina, el primero que los menciona y establece es Varrón, que realiza un tratamiento en cierta manera contradictorio al señalar la serie de tres como la teóricamente natural (por analogía con el comparativo, sin duda), pero la serie de dos (primitivo y diminutivo) como la más frecuente en el uso, admitiendo en todo caso que la multiplicación de formas tenía una motivación semántica, es decir, dependía de la posibilidad de establecer grados (tamaños) en la realidad.

Donato, Consencio y, quizás también, Diomedes establecen los tres grados del diminutivo, pero, a diferencia de Varrón, no observan entre ellos una aminoración semántica, sino una acumulación de sufijos diminutivos, probablemente porque reconocían en la propia lengua que la analogía de los grados del comparativo y del diminutivo se establecía solo en el plano formal y no en el semántico, como más tarde señalará expresamente Macrobio. 
Algunos de los comentaristas de Donato no comprendieron cabalmente el pasaje donatiano comentado acerca de los tres grados del diminutivo, y realizaron una interpretación semántica de lo que en origen era una comparación estrictamente formal. En ellos hay un interés por fijar un límite a la disminución. Prisciano permanece ajeno a la doctrina de los grados y explica el diminutivo en términos similares a los que se leen en Dionisio Tracio y sus escolios.

Todo ello es prueba de la complejidad de la tradición gramatical antigua, en la que, dentro de una uniformidad de temas y taxonomías, los diferentes autores seleccionan, interpretan y comentan según sus intereses, su capacidad y su destreza.

\section{BIBLIOGRAFÍA}

Adams, J. N. 1995: Pelagonius and Latin Veterinary Terminology in the Roman Empire, Leiden.

Baldi, P. 2002: The Foundations of Latin, Berlín-Nueva York.

Barwick, K. 1922: Remmius Palaemon und die römische Ars grammatica, Leipzig. Barwick, K. (ed.) 1964: Flavii Sosipatri Charisii artis grammaticae libri V (reimpr. con addenda et corrigenda de F. Kühnert), Leipzig.

Bécares Botas, V. 1985: Diccionario de terminología gramatical griega, Salamanca.

Bécares Botas, V. 2002: Dionisio Tracio. Gramática. Comentarios Antiguos. Introducción, traducción y notas, Madrid.

Bölte, F. 1886: De artium scriptoribus Latinis quaestiones, Bonn.

Bonnet, G. (ed.) 2005: Dosithée. Grammaire latine, París.

Booij, G. 2000: «Inflection and derivation», en Booij, G., Lehmann, C. y Mugdan, J. (eds.), Morphologie: Ein internationales Handbuch zur Flexion und Wortbildung, vol. 1, Berlín-Nueva York, pp. 360-369.

Chantraine, P. 1979: La formation des noms en grec ancien, París.

Cuzzolin, P. y Lehmann, C. 2004: «Comparison and gradation», en Booij, G., Lehmann, C., Mugdan, J. y Skopeteas, S. (eds.), Morphologie. Ein internationales Handbuch zur Flexion und Wortbildung, vol. 2, Berlín, pp. 1212-1220.

Dahlmann, H. (ed.) 1940: De lingua Latina, Buch VIII, Berlín.

Dammer, R. 2001: Diomedes grammaticus, Tréveris.

Dezotti, L. C. 2011: Arte menor e Arte maior de Donato: tradução, anotação e estudo introdutório. Disertación de Maestría, Faculdade de Filosofia, Letras e Ciências Humanas, Universidad de São Paulo, São Paulo. <http://www.teses.usp. br/teses/disponiveis/8/8143/tde-22092011-161749/>. (25/09/17).

Dressler, W. U., Dziubalska-Kołaczyk, K., Gagarina N. y Kilani-Schoch, M. 2015: «Reduplication, Repetition, Hypercharacterization, and Other Affix-Doubling in 
Child Language», en Manova, S. (ed.), Affix Ordering Across Languages and Frameworks, Oxford, pp. 259-275.

Flobert, P. 1989: «La dérivation nominale chez les grammairiens romains», Latomus 48, pp. 741-752.

Fournier, N. 2013: «Le traitement de la comparaison dans les grammaires du français des XVI ${ }^{\mathrm{e}}$ et XVII ${ }^{\mathrm{e}}$ siècles», Histoire Épistémologie Langage 35.2, pp. 97-132.

Fruyt, M. 2005: «La (dés)-intensification dans les adjectifs latins: les morphèmes de degré», Journal of Latin Linguistics 9 (1), pp. 231-243.

Fruyt, M. 2013: «Temperature and Cognition in Latin», Revue de Linguistique Latine du Centre Alfred Ernout. De Lingua Latina 9: Varia, < http:/www.parissorbonne.fr/IMG/pdf/Temperature-m_fruyt.pdf >, pp. 1-34. (25/09/2017).

Garcea, A. 2012: Caesar's De analogia. Edition, Translation, and Commentary, Oxford.

Goetz, G. y Schoell, F. (eds.) 1910: M. Terenti Varronis De lingua Latina quae supersunt, Leipzig.

Hakamies, R. 1951: Étude sur l'origine et l'évolution du diminutif latin et sa survie dans les langues romanes, Helsinki.

Hanssen, J. S. Th. 1951: Latin Diminutives: A Semantic Study, Bergen.

Haverling, G. V. M. 2011: «La suffixation “diminutive” du latin préclassique et classique au latin tardif», en Fruyt, M. y Spevak, O. (eds.), La quantification en latin, París, pp. 225-257.

Hilgard, A. 1901: Scholia in Dionysii Thracis Artem grammaticam, Leipzig.

Holtz, L. (ed.) 1981: Donat et la tradition de l'enseignement grammatical. Étude sur l'Ars de Donat et sa diffusion (IVe-IXe siècle) et édition critique, París.

Iso, J. J. 1997-1998: «Derivación y composición: su posición en el De lingua Latina de Varrón», Voces 8-9, pp. 57-73.

Keil, H. (ed.) 1961 [1857-1870]: Grammatici Latini, 8 vols., Leipzig.

Kent, R. G. (ed.) 1938: Varro: De Lingua Latina. With an English translation by Roland G. Kent. 2 vols., Londres.

Lallot, J. 19982: La grammaire de Denys le Thrace, París.

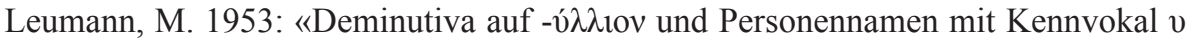
im Griechischen», Glotta 32, pp. 214-225.

Leumann, M. 1977: Lateinische Laut- und Formenlehre, Múnich.

López Gregoris, R. 2005: «El uso del diminutivo en el lenguaje técnico latino», RELat 5, pp. 75-96.

López Gregoris, R. 2016: «El diminutivo latino: entre la denotación y la connotación», en García-Hernández, B. y Penas Ibáñez, M. A. (eds.), Semántica latina y románica. Unidades de significado conceptual y procedimental, Nueva York, pp. 177-198.

Manfredini, A. 2007: «Cuantificación de grado y comparación: paradigmas flexivos y semántica en De Lingua Latina de Varrón», en De Santis, G., Mié, F. y 
Veneciano, G. (eds.), Prácticas discursivas en la antigüedad grecolatina, Ordia Prima Studia 4, Córdoba.

Passalacqua, M. (ed.) 1984: Tre testi grammaticali Bobbiesi (GL V 555-566; 634654; IV 207-2I6 Keil), Roma.

Peppler, C. W. 1902: Comic Terminations in Aristophanes and the Comic Fragments, Baltimore.

Real Academia Española 2009: Nueva gramática de la lengua española, Madrid.

Schad, S. 2007: A Lexicon of Latin Grammatical Terminology. Studia Erudita, 6. Pisa/Roma.

Stock, C. (ed.) 2005: Sergius (Ps.-Cassiodorus) Commentarium de oratione et de octo partibus orationis Artis Secundae Donati. Überlieferung, Text und Kommentar. Sammlung wissenschaftlicher Commentare, Múnich-Leipzig.

Strodach, G. K. 1933: «Latin Diminutives in -ello/a- and -illo/a-. A Study in Diminutive Formation», Language 9 (1), pp. 7-98.

Tarriño, E. 2011: «Comparative clauses», en Baldi, P. y Cuzzolin, P. (eds.), New Perspectives on Historical Latin Syntax, Volume 4: Complex Sentences, Grammaticalization, Typology, Berlín-Boston, pp. 373-426.

Uhlig, G. 1881: Appendix artis Dionysii Thracis, Leipzig.

Uhlig, G. (ed.) 1883: Grammatici Graeci I,1, Leipzig.

Vaahtera, J. 1998: Derivation. Greek and Roman Views on Word Formation, Turku.

Viti, C. 2014: «Latin parts of speech in historical and typological context», Journal of Latin Linguistics 13 (2), pp. 279-301.

Zucchelli, B. 1969: «Sull' origine della funzione diminutiva del suffisso -lo- in latino», Studi linguistici in onore di V. Pisani, Brescia, pp. 1075-1100.

Zucchelli, B. 1970: Studi sulle formazioni latine in -lo- non diminutive e sui loro rapporti con i diminutivi, Parma.

Fecha de recepción de la primera versión del artículo: 04/10/2017

Fecha de aceptación: 14/12/2017

Fecha de recepción de la versión definitiva: 20/12/2017 